\title{
Title: Engineered models of metastasis with application to study cancer biomechanics
}

\author{
Michelle B. Chen ${ }^{1}$, Roger D. Kamm ${ }^{1,2, ~ *}$, Emad Moeendarbary $2,3, *$ \\ ${ }^{1}$ Department of Mechanical Engineering, Massachusetts Institute of Technology, Cambridge, MA, 02139 \\ ${ }^{2}$ Department of Biological Engineering, Massachusetts Institute of Technology, Cambridge, MA, 02139 \\ ${ }^{3}$ Department of Mechanical Engineering, University College London, London WC1E 6BT, United Kingdom \\ *Corresponding authors: rdkamm@mit.edu and e.moeendarbary@ucl.ac.uk
}

\begin{abstract}
Three-dimensional complex biomechanical interactions occur from the initial steps of tumor formation to the latter phases of cancer metastasis. Conventional monolayer cultures cannot recapitulate the complex microenvironment and chemical and mechanical cues that tumor cells experience during their metastatic journey, nor the complexity of their interactions with other, noncancerous cells. As alternative approaches, various engineered models have been developed to recapitulate specific features of each step of metastasis with tunable microenvironments to test a variety of mechanistic hypotheses. Here the main recent advances in the technologies that provide deeper insight into the process of cancer dissemination are discussed, with an emphasis on threedimensional and mechanical factors as well as interactions between multiple cell types.
\end{abstract}

\section{Keywords}

Cancer metastasis, mechanobiology, tumorigenesis, intravasation, extravasation, cell migration, vascularization, cancer models, microfluidics, tumor spheroids

\section{Introduction}

\subsection{Biomechanics of metastasis}

Metastasis is a leading cause of cancer mortality involving a complex multistep process typically encompassing (i) malignant transformation of the primary tumor and acquisition of an invasive phenotype causing tumor cells to disperse and invade the local tissue, (ii) transendothelial migration of cancer cells to enter the blood or lymphatic microvasculature (intravasation), (iii) circulation and survival of tumor cells in the vascular system, (iv) adhesion or physical trapping in a remote microvascular network, (v) transendothelial migration from the vessel lumens into the surrounding tissue, (extravasation), and (vi) subsequent invasion and proliferation in a distal organ to form a secondary tumor (colonization). In addition to a multitude of complex genetic and biochemical cues that involve at all stages of the metastatic cascade, mechanics and mechanical signaling are also playing an apparent role at all steps; the primary tumor, formed as the result of oncogenic mutations and epigenetic cues that lead to disruption in key physiological cellular processes such as cell cycle, is both a biochemically and mechanically abnormal environment (1). While complex biomechanical signaling is involved during tumor initiation and progression, which ultimately can perturb the tumor cells to acquire a malignant phenotype, mechanical signals and mechanotransduction processes also influence the normal behavior of cells and can transform tumor cells to exhibit an invasive behavior $(2,3)$. Aberrations in cell-cell and cell-extracellular matrix (ECM) adhesions and cytoskeletal remodeling lead to abnormal tumor cell morphology and migratory behavior enabling them to invade and migrate through the ECM at the primary site and ultimately intravasate. Circulating tumor cells (CTCs) then have to withstand forces from blood flow to survive through the vascular system and reach the secondary organ. At the secondary site tumor cells exert mechanical forces to escape from the vasculature and invade the ECM of the distal organ. These mechanical interactions 
during the metastatic journey of tumor cells highlight the importance of studying biomechanics in cancer dissemination $(4,5)$.

\subsection{Engineered models of metastasis}

To develop new and more effective modalities for cancer diagnosis, prevention and treatment, it is essential to gain a fundamental understanding of metastasis from many perspectives including not only the molecular and biochemical bases, but also the key mechanical aspects. While in vivo modeling strategies provide much relevant and useful insight, particularly in the context of drug screening approaches, they largely lack the ability to offer detailed quantitative knowledge of biomechanical processes at the level of a single cell, for example, mainly due to technological limitations or high cost (6). Furthermore, due to the complex nature of in vivo experiments, for example the existence of numerous cells types in interacting organs or tissues, it is extremely challenging to test and dissect the role of specific pathways and processes in cancer metastasis. More strikingly, it is increasingly evident that animal models of cancer fail to effectively mimic the underlying molecular mechanisms of cancer initiation and spread in humans (7). Two dimensional (2D) in vitro models that mainly involve culturing a monolayer of cells on flat plastic or glass substrates have served as the standard alternative approaches $(8,9)$. While these $2 \mathrm{D}$ models significantly improved our understanding of the basics of cancer, the lack of the third dimension as well as absence of environmental signals from the stroma in these models, make them unreliable -over $90 \%$ of drugs seemingly effective in these $2 \mathrm{D}$ approaches have been reported to have failed at some stage of human clinical trials $(10,11)$. Indeed, all stages of metastasis inherently involve interactions of tumor cells with the matrix and other cell types in a three dimensional (3D) environment which cannot be recapitulated in conventional monolayer cultures. Inspired by the complex microenvironment that tumor cells experience, and to mimic the biochemical and mechanical cues affecting the dissemination of tumor cells, various engineered models have been developed to recapitulate specific features of each step of metastasis with tunable microenvironments to test a variety of mechanistic hypotheses (12). Here we summarize and discuss some of the main recent advances in these engineered models with the focus on the technologies that provide a better insight into both mechanics and biology of metastatic cancer.

\section{Tumor spheroid models}

The first step of metastasis in most cancer types is the formation of a tumor in a 3D architecture at the primary site. A solid tumor, which from a macro-structural view has a clearly differentiated morphology and increased stiffness compared to its surrounding tissue, is initiated mainly due to disruption in key physiological cellular processes such as cell cycle (13-15). From the microstructural view, the tumor is comprised of cancer cells and a collection of stromal cells such as immune cells, stromal fibroblasts and vascular endothelial cells that are all embedded within the 3D ECM. Tumor growth is maintained via the supply of nutrients and oxygen provided by the vascular system and later boosted via development of new vessels by angiogenesis (16). During the course of tumor development multiple biochemical and mechanical cues distributed in the 3D environment regulate cell-cell and cell-ECM interactions in 3D space leading to creation of a biochemically and mechanically abnormal tumor microenvironment which promotes cancer cell evolution, and often, invasion (4).

The complex microenvironment of native solid tumor presents different cell types organized in a 3D conformation, numerous soluble factors (nutrients, oxygen and growth factors) with 3D gradient profiles and structural/mechanical heterogeneity, which are among the major factors that cause conventional $2 \mathrm{D}$ cultures to be ineffective in recapitulating in vivo conditions $(17,18)$. Spherical 
tumor models represent an alternative in vitro approach capable of better mimicking the 3D structural, cellular and biochemical heterogeneity of tumors $(19,20)$. Tissue engineering techniques have been developed to grow and transform varieties of cellular and extracellular constituents into 3D spherical shapes that are better suited to replicate tumor morphology, growth and microenvironmental factors at different stages of tumor development, such as avascular, vascular, and metastatic disease (21).

\subsection{Avascular tumor models}

Conventional methods of making avascular multicellular tumor spheroids are based on three main principles to aggregate cancer cells into self-assembled spheroids: (i) maintain cells in suspension and (ii) promote cell-cell contacts while (iii) minimizing cell-substrate attachment. For example, in the spinner culture or rotating wall vessel methods the suspension of cells is maintained by continuous stirring of the medium that also promotes cell collision and spontaneous aggregation. Hanging-drop methods and cultures in non-adhesive-wells are other examples of techniques that do not require the application of active forces and are based on maximizing cell-cell contact by trapping cells in a non-adhesive environment and driving cell aggregation via gravity. We refer to (22-25) for extensive recent reviews of methods for making tumor spheroids.

Almost all spherical tumor models exhibit a more realistic response to chemotherapeutics or irradiation, compared to 2D monolayer assays, which make them well-suited for drug discovery and testing of treatment strategies $(26,27)$. However, from the biomechanical perspective, some methods have critical limitations $(23,25)$. For example, while spinner or rotary culture systems are easy to implement (without the need for specialized tools or instrumentation), can be used in high throughput studies, and are capable of generating a ranges of homotypic and heterotypic spheroids, it is challenging to control the size and monitor temporal evolution of individual spheroids making these methods less attractive for investigation of tumor biomechanics or time-evolution of size, morphology or biological behavior. Indeed, the tumor size and growth rate are fundamental biomechanical parameters. The temporal evolution of avascular tumor size normally shows a fast semi-exponential behavior initially followed by a linear regime and slow plateau to reach the final size (28). The growth and stability of tumors rely on the availability of oxygen, nutrients, and metabolites at different radial positions within the tumor, dictated by their diffusion rates that depend upon the radial permeability of the tumor and its microenvironment. Similarly, the effectiveness of drug transport mainly relies on the tumor size and the drug diffusibility (29). Another biomechanical shortcoming of techniques that are based on active forces to keep cells in suspension, is the high level of shear forces that can significantly perturb the growth, morphology and stability of tumor spheroids.

Recent microtechnologies for microprinting of biomaterials and fabrication of microfluidic devices, have shown a promising new avenue in the field of in vitro modeling of cancer progression. Fabrication of 3D microwells and micropatterns using non-adhesive materials offers unprecedented geometric control for the production of tumor spheroids with controllable size and shape $(30,31)$. Microfluidics represent another microtechnology that allows continuous perfusion, precise application of flow and gradients of different soluble factors (32) in addition to confining cells within small channels/wells or hydrogels, to facilitate controlled self-assembly. While 3D-bioprinting of cells represents a useful new approach to patterning of cells in precise 3D architectures, care must be taken to avoid exposure of cells to, for instance, high levels of pressure or heat (33). The temporal evolution of spheroids made in these microfabricated and bioprinted systems can be imaged live from the early stages (self-assembly of cells) and the biophysical changes (such as cellular growth rate and morphology) in response to different drugs, antibodies and growth factors can be monitored in fine detail through to the development of necrotic core and tumor growth cessation. 


\subsection{Matrix-embedded and vascularized tumor models}

Alterations in cellular processes at the single cell level, such as uncontrolled cell proliferation, inappropriate cell survival, and specific initial cell-cell interactions such as those occurring in multicellular tumor spheroids, are the initiators of tumor formation. However, cells present within the tumor dynamically interact with ECM components and other cells within and surrounding the tumor and these cell-cell and cell-ECM interactions contribute to the tumor morphology and growth and the possible transformation of tumor cells to a malignant phenotype. Indeed, tumor growth exerts forces against the surrounding ECM, which mainly consists of proteoglycans and glycosaminoglycans, collagens, fibrin, and fibronectin, and leads to an excessive build-up of stress within the tumor, which potentially influences tumor cell phenotype (34-36). Therefore, while suspended (contact inhibited) spheroids are a vast improvement to 2D monolayer cultures, they still have limited ability to mimic biomechanical features of native tumors (37). Matrix/hydrogel embedded spheroid models therefore improve the ability to mimic tumor growth and are capable of better capturing cell-ECM interactions, influences of physical boundaries, protrusion formation and detachment, and ultimately invasion of tumor cells. The mechanosensitive behavior of tumor spheroids in these systems can be investigated via tuning structural and mechanical properties of the matrix for example by changing the polymer concentration or degree of cross-linking (38). Furthermore, in these systems cell-induced changes in the bio-mechanical properties of the ECM (such as matrix deposition and active contraction ) around the tumor, which also influences diffusion gradients (39), can be investigated.

Co-culture of matrix embedded tumor spheroids with other cell types and application of fluid flow and chemical gradients are essential factors in mimicking native tumor microenvironment that are now possible in emerging lab-on-chip technologies (40). For example, mechanobiological interactions between tumor cells and cancer associated fibroblasts (CAFs) promote tumor invasion and metastasis $(41,42)$ and a recent microfluidic platform enabled the study of reciprocal signaling between collagen-embedded tumor spheroids and CAFs cultured in proximity of spheroids (43). In addition to providing precise spatiotemporal control of growth factor gradients, temperature, interstitial flow and interaction with multiple cell types and ECMs, microfluidic devices have been also employed to regulate oxygen tension within the artificial tumor environment around tumor spheroids grown in 3D (44) or 2D culture of cancer cells (45), impacting tumor vascularization as well as cancer and stromal cell motility.

Beyond some critical size ( $>1 \mathrm{~mm})$, diffusion mechanisms for supplying oxygen and soluble factors into the tumor become insufficient to maintain tumor growth and result in the development of a necrotic/hypoxic region at the core surrounded by a highly proliferative outer rim. Therefore, the avascular tumor employs robust vascularization mechanisms to boost its growth via enhanced delivery of different factors, especially oxygen. Vascularization of the tumor and its immediate vicinity is initiated through the recruitment and activation of endothelial cells, mainly triggered by hypoxia activated pathways(46), and progresses and is maintained by signaling with other supporting cells of the tumor microenvironment, such as immune cells, mesenchymal stromal cells and fibroblasts (16). However, the newly 3D formed vascular network is a significant departure from the normal architecture of blood and lymphatic networks, leading to an aberrant interaction between the fluid with solid phase of the tumor, increased interstitial fluid pressure within tumor and persistence in the lack of gas and nutrients (47). Furthermore, in addition to biochemical signals that drive vascularization of the tumor, the growth and architecture of tumor-specific vasculature are highly influenced by physical factors such as mechanical, hydrodynamical, and collective processes (48). 
Early stages of angiogenesis and sprouting can be simply modeled by culturing a monolayer of endothelial cells on top of a thin hydrogel, or more recently vessel-like channels lined with endothelial cells and surrounded by ECM (49), while investigating the ability of endothelial sprouting under different chemical and mechanical angiogenic factors. For example, taking advantage of new microfluidic technologies and novel hydrogels enabled investigation of fluid shear stress (50) or ECM mechanical properties (38) on angiogenic sprouting (51). To provide a more realistic approach relevant to tumor sprouting and angiogenesis and to take into account effects of tumor cells, in other studies endothelial cells were co-cultured with cancer cells separated by a barrier or layer of matrix to avoid direct endothelial-cancer cell contact and induce sprouting under a more complex environment. In these approaches, the effects of different microenvironmental parameters such as structure and stiffness of the ECM and cancer and stromal cell types, on the length of endothelial sprouts and invasion density can be evaluated (52). Interstitial flow and shear stress are other factors that regulate sprouting and have been investigated in detail with microfluidic models(53).

To study the process of vascularization at tumor scale, endothelial cells can be added to the typical spheroid system: for example, by using co-cultures to generate spheroids consisting of a mixture of cancer cells and endothelial cells as well as other stromal cells (54-56) and suspending these in a 3D matrix, or by co-culturing spheroids comprised of cancer cells and other stromal cells (excluding endothelial cells) and suspending them in matrix with endothelial cells embedded inside or seeded on the outside surface of the matrix (57). For example, preformed U87 spheroids were co-cultured with HUVECs that were separated by gelatin-methacryloyl hydrogels allowing non-contact communication and sprouting of HUVECs through the hydrogel (58). Furthermore, using semisynthetic hydrogels of tunable stiffness, ranging from 500 to $3000 \mathrm{~Pa}$, to create sophisticated vascularized cultures of cancer spheroids with endothelial cells and mesenchymal stromal cells (59, 60 ), it is possible to investigate effects of mechanical properties of ECM on the process of tumor angiogenesis.

\section{Models of cell migration and invasion}

While multiple processes are involved during cancer metastasis, invasion of tissue at the primary site is perhaps the most central step in this complex biological phenomenon. From the biomechanics standpoint, the key questions at this step are how biomechanical cues from the microenvironment influence and drive an invasive phenotype and how tumor cells employ robust modes of migration to invade into the interstitial tissue at the primary site. These questions are also relevant at the later steps of metastasis where cancer cells employ similar migration mechanisms to navigate through secondary tissue upon extravasation from the vasculature. To address these issues, a myriad of migration assays has been applied to understand fundamentals of cell migration under the influence of typical chemical and mechanical factors such as chemical gradients, stiffness, topography, stiffness gradients, fluid shear stress and confinement $(61,62)$. In many of these models, the basic regulators of cancer cell motility such as actomyosin contractile forces and adhesion have been studied without explicit inclusion of ECM (63). For example, to evaluate the influence of confinement on cancer cell migration, researchers have turned to microfluidic devices containing narrow constrictions and capable of inducing chemical gradients, to drive cancer cell movement through small channels while probing their dynamics at high spatiotemporal resolution $(64,65)$. Using these devices, intriguing mechanics have been revealed: cancer cells can migrate persistently through narrow constrictions even after some of the basic hallmarks of $2 \mathrm{D}$ cell motility, i.e. actomyosin activity and integrin-mediated adhesion, are disrupted, by relying upon fundamentally different migration mechanisms, such as water permeation $(66,67)$ and blebbing $(68)$, that cancer cells can employ specifically to move through confined spaces. In addition, the importance of 
nuclear deformation, and even mechanical damage to the chromosomes or nuclear envelope, have been characterized during migration through confining microenvironments using conceptually similar microfluidic devices (69).

Despite the successes of these 2D or open-channel experiments devoid of ECM, inclusion of ECM as a major component of cancer microenvironment in in vitro models is a significant step towards achieving more realistic biomechanical models of cancer invasion. Indeed, even "inactive matrices", lacking the capabilities of remodeling and degradation, influence cell migration via their distinct physical, biochemical, and mechanical properties(70). In addition, the ECM in the tumor microenvironment is highly active, undergoes remodeling, changes in composition, and degradation, and its disorganized structure and abnormal biomechanical properties all contribute to the dysfunctional behavior of cancer cells as well as the associated stromal cells. To reconstruct the native ECM, ranges of natural and synthetic biocompatible scaffolds with tunable biochemical and mechanical characteristics such as cell adhesive ability, biodegradability, 3D topography and mechanical stiffness have been engineered. For example, to dissect the role of matrix stiffness and composition on transformation to malignancy, interpenetrating polymer networks of alginate and reconstituted basement membrane matrix that provide a wide range of mechanical properties and control over matrix stiffness independent of adhesion ligand density, have been recently devised(71). While advances in engineering novel hydrogels continue, integration of natural and synthetic hydrogels into in vitro platforms, and particularly microfluidic systems, has resulted in new studies of the dynamics of cancer cell invasion in 3D and under controllable biophysical and biochemical stimuli (32). For example, to study the effect of interstitial flow on tumor cell motility, a pressure gradient had been imposed across a channel filled with collagen hydrogel containing cancer cells, and the dynamics of cell migration under the flow had been quantified (72). Furthermore, using 3D microfluidic chips, it has been shown that breast cancer cells (MDA-MB231) embedded in a collagen gel switch their morphology and mode of migration, from mesenchymal to amoeboid, when challenged by interstitial flow velocities typical of those found in the vicinity of a tumor $(\sim 3-10 \mu \mathrm{m} / \mathrm{s})(73)$ and mechanobiological mechanisms that underlie migration of cancer cells in the upstream direction of flow have been identified(74). Despite the development of several macroscale and microfluidic models, however, which have studied the effects of fluid flow on individual cells(75) or systems of cell aggregates(76) and avascular tumor spheroids $(77,78)$, in vitro models of interstitial flow in vascularized tumors is still lacking. The challenge is to generate a model in which the interstitial flow emanates from leaky vessels within the tumor, and flows outward, influencing transport and cell migration in the tumor microenvironment.

\section{Vascular models (MC)}

It is well known that the progression of metastasis involves complex and direct interactions with the vasculature. Hematogenous dissemination begins with the invasion of tumor cells past the vascular endothelial barrier, followed by its transit in the circulation and finally extravasation from within the blood vessels into the parenchymal space to form distant tumors.

The process of intravasation and extravasation inevitably involves the perturbation of the endothelial barrier through which they traverse, and can be modulated by both heterotypic cell-cell paracrine or contact dependent mechanisms. For instance, secreted factors from tumor and ECs such as transforming growth factor-beta (TGF- $\beta$ ) or vascular endothelial growth factor (VEGF) upregulated in tumor vasculature, can act to decrease endothelial barrier function likely through disruption of cell-cell junctions, and facilitating intravasation(79) (80). Contact interactions such as firm tumor cell-endothelial adhesion via integrins, cadherins, CD44, immunoglobulin (IgG) superfamily receptors or selectins are believed to be the first step in cancer cell extravasation. 
Commonly used in vitro models of tumor cell-endothelial interactions during metastasis involve the use of Boyden chamber/Transwells and inserts lined with a monolayer of endothelial cells on the top or bottom surfaces to study extravasation or intravasation events, respectively. These assays provide a relatively simple and high throughput method for parametric cell migration studies, and ease of quantification of transendothelial migration events. More recently, similar models incorporating a 3D ECM beneath the endothelialized layer have aimed to increase physiological relevance, by mimicking the compliant and soft nature of the subendothelial matrix environment (81). However, in the majority of these assays, direct visualization of tumor-endothelial interactions cannot be achieved due to low optical accessibility, and physiological relevance is limited since planar monolayers on stiff plastic or glass substrates do not recapitulate the mechanical properties of the subendothelial matrix or the $3 \mathrm{D}$ tortuosity and narrow diameters of the in vivo microvasculature.

To address these issues, recent bioengineering advances have allowed for more realistic models of the human microvasculature. For example, lining channels and cavities with endothelial cells can result in networks of vessels. Channels embedded within 3D hydrogels to mimic the ECM can also be achieved using pin-pull out(82), sacrificial gel methods(83) or 3D printing(84)(85). Endothelial cells such as HUVECs can be introduced into the channels to form patent lumen-like structures. Soft lithography approaches also facilitate the formation of interconnected microchannels which mimic a blood vessel network, and allow application of relevant fluid shear stresses and dynamic monitoring of tumor cell adhesion and transmigration $(85,86)$ In fact, some of these vascularized platforms have begun to be applied to study the mechanisms of tumor-endothelial interactions during metastasis. However, in general, monolayer-based systems face several challenges, including geometrical over-simplification, as tumor-endothelial interactions typically occur in complex 3D microvascular beds of 5-30 $\mu$ m diameters, rather than $>100 \mu \mathrm{m}$. Currently, there remain technical challenges in achieving vessel diameters of $<100 \mu \mathrm{m}$ via soft lithography and/or templating approaches.

A more recent methodology for creating vascular models draws on the phenomenon of selforganization. Microvessel networks can be formed via the self-organization of endothelial cells such as human umbilical vein endothelial cells (HUVECs) seeded within 3D collagen type 1 or fibrin matrices. Using this method, an in vitro microfluidics based platform has been developed to recapitulate both the tumor neovascularization and intravasation steps of metastasis. Direct culture of human primary endothelial cells with human tumor cells and fibroblasts results in endothelial tube formation in the direction of the tumor spheroid. Intricate vascular beds appear to form after only 7 days of culture(87). Confocal microscopy in this and similar assays reveal that tumor cells can under trans-endothelial migration (TEM) via the transcellular route(88).

While vasculogenesis-like tube formation in vitro has long been demonstrated in both fibrin droplets and more complex platforms like the ones mentioned above(89), most systems remain un-perfusable due to the lack of accessible entry points into the vasculature. To address this, an in vitro microfluidics-based assay has been developed featuring perfusable human microvasculature, and its utility demonstrated by investigating the extravasation stage of metastasis. A soft lithography PDMS-based approach is used to generate microchannels which house 3D fibrin gels for cell culture. In many of these systems, interspaced upright micro-posts delineate the boundaries of each gel region via surface tension generated at the gel-air interface. HUVECs and supporting stromal cells such as human lung fibroblasts are suspended in fibrin gels and under an optimal combination of growth and stabilization factors, single HUVECs connect with neighboring cells to form patent lumens within 4-5 days. Importantly, characterization of the in vitro vasculature confirmed that endothelial barrier functions were similar to that of in vivo venules and that lumens are positive for 
continuous CD31 and VE-cadherin, and possess the correct polarity of basement membrane proteins including collagen IV, fibronectin, and laminin $(90,91)$. Due to the design of the micro-posts, microvessels are able to form open lumens that connect the inlet and outlet ports of the device with the vascular bed. Contrary to previous platforms of self-organized networks, these devices(92) uniquely allow for the direct perfusion of circulating tumor cells and blood cells into the vasculature, where they can arrest via physical trapping. Dynamics of tumor-endothelial cell interactions can then be visualized with high resolutions using conventional confocal microscopy. For instance, tumor cells are observed to begin the extravasation process by extending thin filopodial-like protrusions past the endothelial barrier, while the remaining portion of the cell body remains largely spherical. This morphological phenotype is dependent on the engagement of tumor beta-1 integrins to subendothelial laminin, and is a prerequisite for successful extravasation. Through detailed confocal microscopy, we have also visualized the disruption of the endothelium at cell-cell junctions, which is followed by subsequent re-sealing after the completion of transmigration.

\section{Studies of intravasation}

Mechanisms of intravasation and whether cancer cells actively or passively migrate through blood and lymph vasculature to enter circulation are under continued debate (93). Some of the most vivid observations were first obtained by intravital imaging in murine models (94). These provided new insights into the process by which tumor cells, often interacting with tumor associated macrophages, are able to migrate toward and cross the endothelial barrier.

Tumor cells gain access to local blood or lymphatic vessels either within the tumor, or in the surrounding tissue, using the various types of migration that have been observed both by intravital imaging (95) and in transwell (12) or recently microfluidic assays (96). When the vessels are in close contact with the tumor, it is believed that clusters of cancer cells can break away from the primary tumor and enter directly into the circulation, as the vascular network is poorly formed, and the barrier posed by the vascular wall is abnormal and highly permeable (97). Alternatively, away from tumor mass, cells can detach from the primary tumor and migrate in single or collective forms through the surrounding tissue where they can encounter a vessel (98). Upon reaching the vessel, they can transmigrate into the lumen, first by passing the basement membrane, then breaching the endothelial barrier. Once the tumor cell (TC) has gained access to the vessel lumen, it experiences fluid shear stress which then causes the cell to detach from the endothelium, thus becoming a circulating tumor cell (CTC) where it experiences a variety of biomechanical stimuli during its transit to the metastatic site.

During both migration through the matrix and intravasation, TCs undergo large deformations and experience stresses often far in excess of those typically found in the host tissue. Indeed, metastatic cells have been found to be both softer (lower shear modulus) and more contractile than their nonmetastatic counterparts (99).To study the biomechanical events associated with intravasation requires comprehensive 3D models since the relevant processes involve multiple cell types passing through complex structures, so our understanding of the mechanisms of intravasation has progressed slowly. In vitro models have since been developed, and used to dissect and better understand the factors influencing intravasation. The simplest of these are transwell or modified Boyden chamber systems with endothelial cells plated onto the bottom of the membrane and tumor cells seeded into the upper chamber, either directly on the membrane or embedded in a hydrogel injected on the membrane. Some early studies examined the TEM of a variety of tumor cell types, and were able to demonstrate a link between changes in monolayer integrity and the rate of transmigration (100). But, while useful, these models suffered from the artifact of the transwell membrane, and had limited imaging capabilities. 
With the advent of microfluidic assays, early intravasation studies were conducted in systems with a monolayer on the side face of a hydrogel, enabling much improved imaging and circumventing the need for an artificial membrane. These studies also demonstrated an important role for tumorassociated macrophages (TAM), demonstrating that one of the mechanisms by which intravasation was enhanced was the increased permeability of the monolayer associated with secretion of TNF- $\alpha$ by the TAMs, augmenting the rate of transmigration nearly 10-fold (96). Relying on high resolution confocal imaging, these experiments were low throughput, however, and given the low rates of intravasation, difficult to use as a basis for studying detailed mechanisms. More recent models have taken advantage of the capabilities to form vessels within a gel matrix, either by casting around a rigid rod and seeding endothelial cells in the lumen to form a vessel (101), or by growing networks via a vasculogenesis-like process, as described in Section 4. Using the casting method, Wong and Searson observed multiple types of EC-TC interactions including migration to the abluminal surface of a vessel, intravasation, and induction of sprouts from the larger vessel toward the tumor clusters.

In a recent study, Ehsan and co-workers developed a system in which they formed tumor spheroids seeded with endothelial cells, suspended them in a fibrin gel containing fibroblasts, and injected the resulting solution into a microfluidic device (87). The ECs were observed to form vascular networks both inside of and sprouting into the gel surrounding the tumor spheroids. With this assay, they observed tumor cells inside of the vessel lumens, and that the frequency of these observations increased under hypoxic conditions. And while the vessels in this experiment were not perfusable, other, more recent work published by Nashimoto et al., have now shown that it is possible to link an intra-spheroid vascular network with an external one (sprouting into the gel external to the spheroid), enabling the vessels to be perfused from side media channels (102). This now raises the prospect of an even more realistic model of intravasation for future study.

\section{Cell plugging or adhesion to the vascular wall}

Extravasation involves a cascade of events consisting of tumor cell arrest on the endothelium resulting in the formation of dynamic contacts that give rise to significant cytoskeletal changes, followed by tumor cell transendothelial migration (TEM) and subsequent invasion. There have been two general hypotheses regarding the mode of arrest of CTCs in a blood vessel: (1) mechanical trapping of cells and/or (2) active preferential adhesion of the tumor cell onto the endothelium at a distant organ. James Ewing proposed that preferential metastasis to specific distant organs is dictated by the anatomy of the blood and lymphatic vessels and blood circulatory paths between primary and secondary tumor sites (103). CTCs are relatively large ( 20 micron) in diameter and may therefore become physically trapped in small vessels of the microcirculation, become activated, and eventually transmigrate(104). However, Steven Paget's "seed and soil" hypothesis suggests that there exist interactions between different tumor cell types and specific organ microenvironments that guide their metastatic spread. The vasculature of such organs may be primed with surface receptors/molecules or secrete chemokines that cause specific tumor cell types to preferentially "home and seed" at that particular tissue environment. For instance, breast cancer cells frequently metastasize to the bone(105). Although two distinct phenomena have been described, is it clear now that the two theories may not be mutually exclusive $(106,107)$.

Active adhesion: It is believed that active adhesion to the endothelium is required for tumor cell arrest. While leukocytes are known to roll and arrest on the endothelium, the same phenomena has not been observed for cancer cells in vivo. However, cancer cells have been shown to roll on and arrest on planar monolayer models, and this appears to be dependent on selectin ligands including those for E-selectin(108, 109). Furthermore, the expression of E-selectin on ECs and its ligands on tumor cells appear to be critical for efficient metastatic progression, such as in the case of bone marrow homing in vivo(110). Other recent studies have shown that CTCs contain microtentacles 
that may be instrumental in their attachment to the endothelium or aggregation in the circulation (111). In a microfluidic endothelial layer model developed by Shin et al, it was found that following intravasation, tumor cells adhered and arrested on the endothelium and that E-selectin expression dictated the level stable attachment of tumor cells under shear flow conditions(86). However, Eselectin is not normally expressed on quiescent ECs, but usually induced by inflammatory cytokines. In the case of metastasis, it is likely that tumor cells themselves can secrete such cytokines, as shown by the increase in E-selectin foci in the mouse lung after perfusion with tumor cell conditioned media(112). Furthermore, the presence of tumor cells has been shown to induce E-selectin expression in liver endothelium through the indirect action of tumor-recruited macrophages(113).

In addition to initial rolling, tumor cells may also form stable adhesions with the endothelium through the action of integrins, CD44, and MUC1(108). CD44 has been extensively studied as a primary tumor cell ligand for selectins during metastatic progression. For instance, CD44 mediates the adhesion of prostate and breast cancer cells to the endothelium, and facilitates transendothelial migration(114). Importantly, activation and engagement of CD44 with endothelial ligands can further lead to downstream changes in gene expression, such as the up regulation of integrins $\beta 1$ and $\beta 2$, which could enhance transendothelial migration efficiency(115).

It has been shown that integrins including $\beta 1, \beta 4$ and $\alpha v \beta 3$ can modulate the adhesion of prostate cancer cells to an endothelial monolayer in vitro. Unlike E-selectin, which typically requires stimulation for expression, integrins often do not require the presence of exogenous chemokines for their activation(110). It is interesting to note however, that findings on an in vitro monolayer and using microvascular models suggest that while $\beta 1$ integrins do mediate stable intraluminal arrest, the engagement of active tumor $\beta 1$ is not primarily with the endothelium, but rather the subendothelial matrix. Detailed confocal microscopy shows that the majority of tumor cells remaining adhered to the intraluminal surface had already made small protrusions, which contacted the basement membrane(116). Regardless, it is likely that multiple adhesion receptors on tumor cells can mediate their adhesion to the endothelium, and may also depend on the phenotype of endothelial cells at the site of arrest.

Physical trapping: Video microscopy shows that tumor cells slow down and arrest in narrow capillaries in zebrafish and CAM embryo models immediately upon injection, suggesting that cells are first physically restricted, and may subsequently form weak and then stable attachments to the endothelium or underlying basement membrane. Furthermore, tumor cells are found to be arrested in the narrow capillaries of the lung post tail-vein injection in mice(117), and have only been observed to arrest in larger diameter vessels ( 50-100 microns) in hepatic sinusoids, which express E-selectin. In microvascular models of extravasation, perfused tumor cells are most often found to flow and decelerate in narrow capillaries before eventually arresting, in a matter of a few seconds. HUVECs in this system do not appear to express high levels of E-selection (before or after tumor cell perfusion) and competitive blocking of E-selectin with antibodies does not alter the rate of tumor cell trapping in the vascular bed (unpublished results). Importantly, no changes in transendothelial migration rates of tumor cells were found when blocking E-selectin, suggesting that selectindependent tumor-endothelial interactions is not a requirement for tumor cell arrest nor transmigration. However, the necessity of these adhesive interactions may be organ site dependent.

\section{Extravasation at the distant site}

\subsection{Role of endothelial dynamics, basement membrane and subendothelial matrix}


Endothelial dynamics: While tumor cells are highly dynamic in terms of migration and morphology during transendothelial migration, endothelial cells are also highly motile. Rather than being a passive barrier to tumor cells, they are highly contractile, migratory, and remodel quickly after tumor cell penetration. It has been shown that tumor cell extravasation is correlated with endothelial monolayer barrier function. For instance, treatment of microvasculature with $50 \mathrm{ng} / \mathrm{mL}$ TNF- $\alpha$ resulted a significant increase in vascular permeability, as well as extravasation potential of MDAMB-231 cells(90). Interestingly, the same behavior is observed in an in vitro model of intravasation. Tumor cells are also often found to transmigrate through the paracellular route, with transcellular extravasation not yet observed in vitro. Paracellular migration is most often achieved at tri-cellular junctions in endothelial monolayers, where the junctional adhesions are frequently found to be weaker.

Basement membrane and the subendothelial matrix: The role of tumor-matrix interactions at the primary tumor site has been well studied. In particular, the expression and up-regulation of proteins such as tumor integrins and protease secretion including MMPs are key for modulating the invasive potential of tumor cells, and have been well characterized(118-121). However, the molecular players modulating tumor-matrix interactions at the distant metastatic site are less clear, as it now involves crossing of the endothelial barrier and interactions with the vascular basement membrane. Recent work has shown that adhesion of tumor cell $\alpha 3 \beta 1$ and $\alpha 6 \beta 1$ to subendothelial laminin is involved in successful transmigration. In particular, activated $\beta 1$ integrin engagement is required for stable protrusion formation past the endothelium, which is followed by F-actin recruitment to the protrusion tip and translocation of the tumor cell past the EC barrier likely via acto-myosin contractility(116).

\subsection{The role of stromal cells and local immune cells}

Metastasis is a function not only of tumor cells, but involves inhibitory and cooperative interactions with normal host cells. This includes platelets, a wide range of immune cells, such as monocytes, macrophages, neutrophils natural killer (NK) cells, as well as stromal cells including fibroblasts and pericytes. While tumor cells at the distant site may have acquired necessary traits to complete the metastatic cascade as early as the primary tumor, it is highly likely that the host microenvironment at the secondary site plays a large role in shaping its final metastatic potential. For the case of extravasation, it has been observed that host immune cells such as platelets and neutrophils can interact with tumor cells in the bloodstream during the circulation and extravasation stages. During the beginning phases of transit and arrest, tumor cells generally remain exposed to blood flow, and are thus prone to death induced by excessive shear stress, or by the action of clearing immune cells such as NK cells. Thus, protection of tumor cells from these environmental stresses could increase their rate of retention as well as survival, at the secondary site. In this respect, it has been found that tumor cell transit can activate the coagulation process, resulting in the formation of tumor-platelet clusters $(122,123)$. In vivo, coagulation and platelet activation can enhance metastatic spread (124). In vitro, it has been found that a simple co-culture on plastic of quiescent platelets with breast cancer cells MDA-MB-231 can result in platelet aggregation in a matter of minutes, and that the same behavior occurs in in vitro microvascular networks. In fact, tumor cells are often found to be arrested in contact with platelet clusters, and this this correlated with increase tumor cell retention rate as well as increased transmigration rates (unpublished findings). This is in line with previous findings showing that $\alpha v \beta 3$ on tumor cells and $\alpha$ II $\beta 3$ on activated platelets can bind together via soluble fibrin found in the plasma, and that genetic elimination of $\beta 3$ integrins on platelets results in a defect in metastasis(123). It is possible that clustering results in tumor cell shielding from shear stress and NK cell tumorilytic activity, and enhance embolus formation and tumor cell intravascular arrest. In addition to enhancing arrest and survival, tumor-activated platelets can release adenine nucleotides, which act to induce the opening 
of the endothelial barrier and thus facilitating extravasation. This is dependent on the endothelial $\mathrm{P}_{2} \mathrm{Y}_{2}$ receptor, which is activated by platelet-derived ATP(125).

Intravascular interactions with neutrophils have also recently been shown to play a role in extravasation. Leukocytes can play cooperative roles in enhancing the early stages of metastasis, as evidenced by the impaired tumor cell seeding when tumor-neutrophil interactions were attenuated in L-selectin -/- mice(126). Furthermore, tail-vein injections of human neutrophils 1 hour after injection of melanoma cells results in increased tumor cell retention in the lung and metastatic foci formation(127). Extensive studies by the Dong group has shown that tumor cells can attract and activate neutrophils through tumor-derived IL-8, and that this upregulates the expression of CD11b and $\beta 2$ integrin on neutrophils. This enhances the adhesion between tumor cells, neutrophils and the endothelium through ICAM-1, and ultimately results in increased tumor cell arrest and resistance to shear stresses (128). Recent work in our lab using on-chip microvasculature has not only confirmed these results, but also shown that systemically inflamed neutrophils can affect the extravasation potential of tumor cells through IL-8 dependent mechanisms in a different manner. While TCs and LPS stimulated polymorphonuclear neutrophils (PMNs) ) first arrest intraluminally in heterotypic clusters similar to those observed in vivo (129), studies have also focused on the characterization of dynamic PMN-TC-endothelial interactions after cluster arrest. These have shown that clusterassociated PMNs are not statically aggregated with TCs, but migrate in a confined manner near the original TC-PMN cluster. This behavior is attributed to chemotactic confinement mediated by PMN self-secreted IL-8 and tumor-derived CXCL-1, and enhanced by the endothelial glycocalyx, ultimately resulting in further sequestration of PMNs near entrapped tumor cells for $>6$ hours. The significance of this lies in the positive correlation between increased extravasation potential and physical proximity of TCs from PMNs. This unique mode of PMN autologous chemotaxis dependent on initial TC-PMN clustering appears to enable the spatial localization of proextravasation factors such as IL-8, which plays an additional role in facilitating transendothelial migration of adjacent TCs in the clusters, through disruption of the endothelial barrier.

Beyond the endothelium lies a plethora of host cells which may directly or indirectly affect the extravasation potential of tumor cells. In the microvasculature, predominant stromal cells include fibroblasts and pericytes, which are responsible for the stability of the extracellular matrix through both their physical presence and secretion of diverse proteins. The essential contribution of pericytes to vasculature development and maintenance has long been known - they participate in the regulation of blood flow and vessel permeability, as well as vascular wall stabilization(130). Since extravasation efficiency has been found to correlate with vascular barrier function, it is likely that the presence of pericytes may play active roles in modulating transendothelial migration. In support of this hypothesis, we have observed the co-culture of HUVECs with human placental pericytes in our microfluidic platforms can result in the formation of perfusable microvascular networks sheathed periodically with pericytes on the abluminal surfaces. Interestingly, extravasation rates from pericyte-covered vasculature were dramatically attenuated compared to mono-cultured systems. 


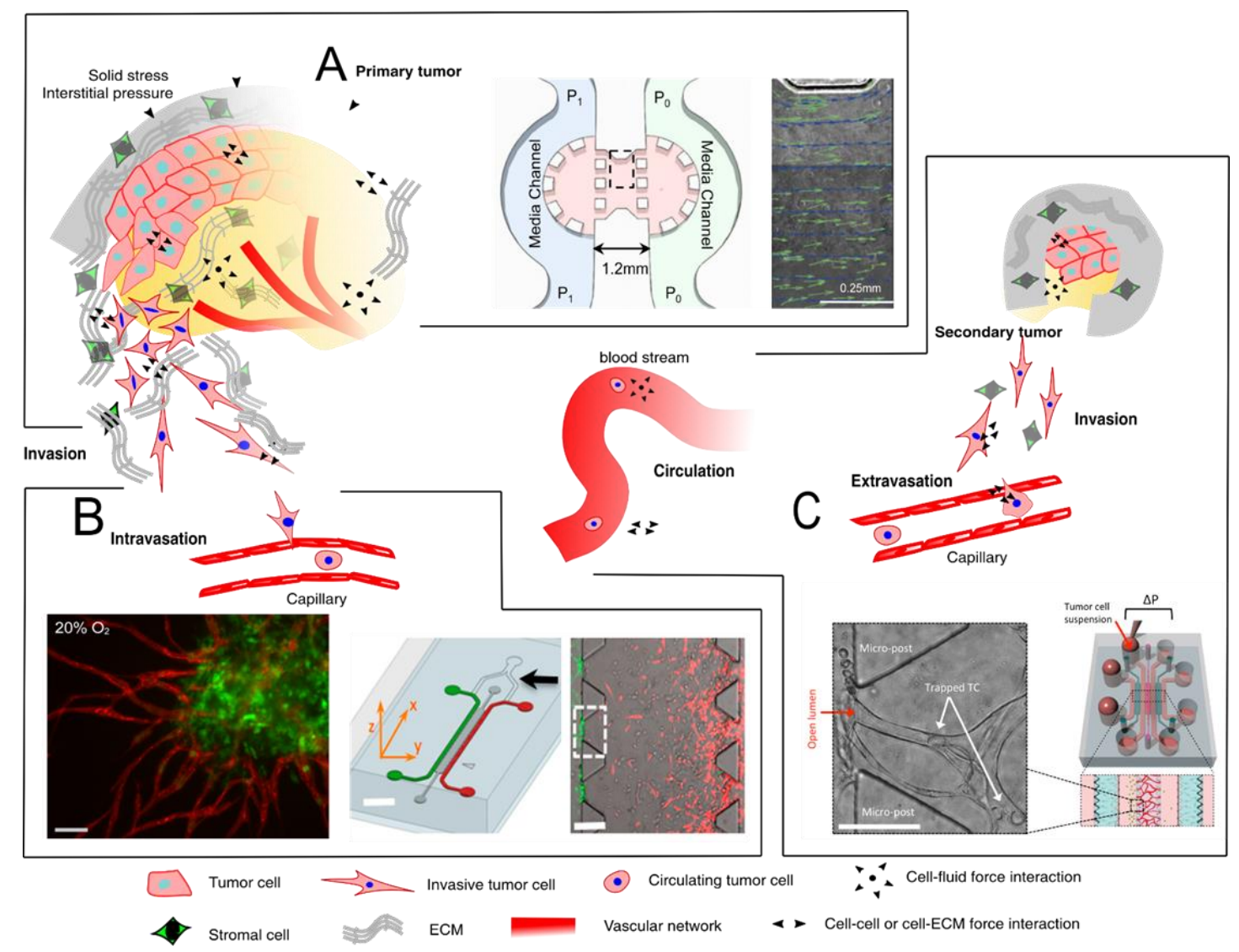

Figure 1. Overview of models of the metastatic cascade. (A) Tumor cells at the primary site are subject to various mechanical perturbations such as stiffening of the extracellular matrix and interstitial fluid pressure. Microfluidic models have been developed to mimic interstitial fluid flow through a 3D matrix while being able to observe the dynamics of tumor cell migration in response to this mechanical stress(72). (B) Tumor cells enter the circulation via a process called intravasation, where cell migrate from the parenchyma and past the endothelial barrier. In vitro models include self-assembled capillary beds around tumor spheroids(87), and endothelial monolayer devices that allow for direct observation of transmigration events at high resolutions(96). (C) Circulating tumor cells eventually arrest and exit the vasculature via extravasation into the surrounding vascular basement membrane and ECM. This process has been modeled in parallel flow chamber devices with endothelial monolayers $(128,131)$, as well as self-assembled vascular beds in 3D hydrogels, through which tumor cells are perfused and migration behavior observed in real-time(132). 
A
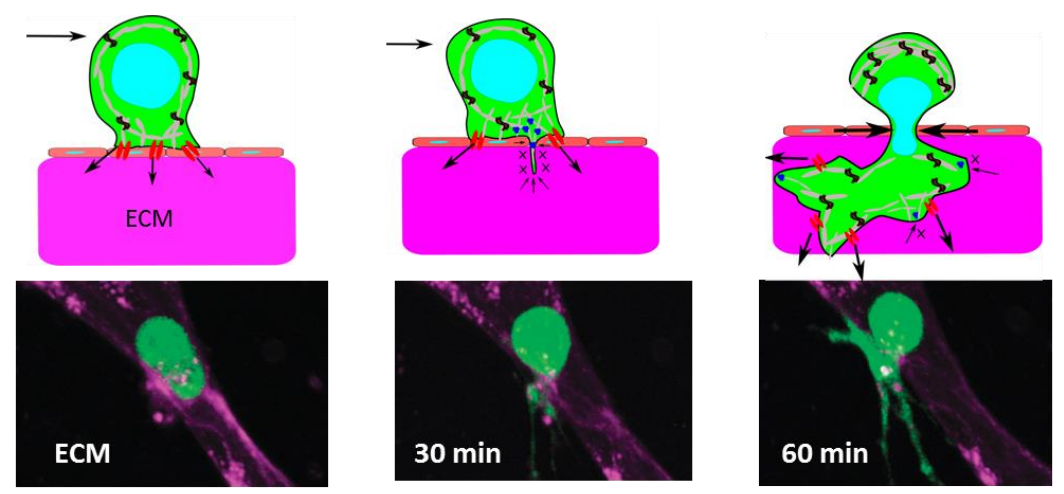

actomyosin complex
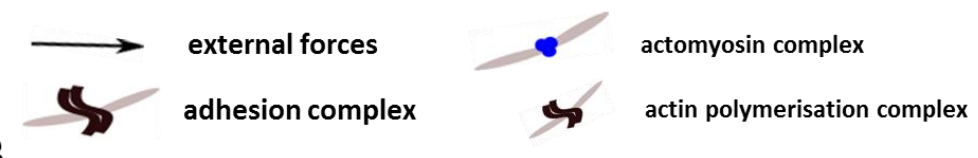

B
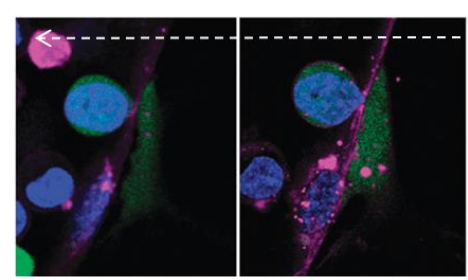

$\leftarrow---\stackrel{20 \mathrm{~min}}{-}$
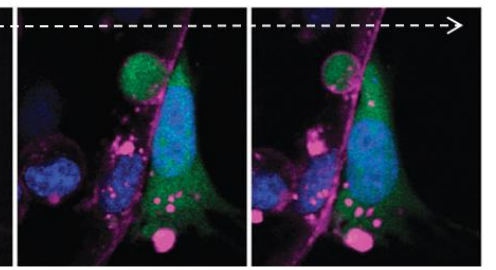

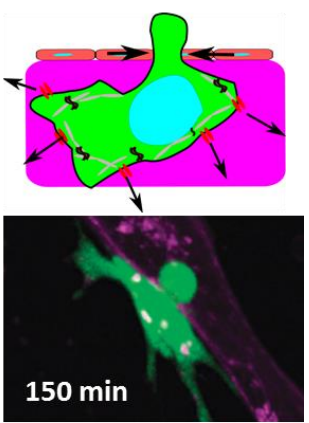

matrix/ EC proteinases

Figure 2. Dynamics of tumour cell extravasation. Based on recent experimental observations, two major processes suggested to be involved in extravasation of CTCs: In the first step CTCs (singly or in aggregates) arrest on the vascular endothelial cells (ECs) by either being trapped inside microvasculature (physical occlusion) or forming transient dynamic bonds or firm attachments with ECs. Following arrest, the CTC establishes firm attachments through clustering of persistent adhesion molecules in order to spread on the surface of ECs and create strong anchors essential for cytoskeletal rearrangements and transmigration. The second step is transendothelial migration (TEM) in which the TC transmigrates through the endothelial barrier by sending out dynamic protrusions that penetrate across the EC (primarily via paracellular routes). Upon reaching the basement membrane ECM, the protrusion adhere and actomyosin contractility pulls the cell across the EC monolayer into the underlying tissues. (A) High resolution time lapse imaging revealed that after tumour cell is arrested on the endothelium it sends out thin fillipodial-like protrusions that extend across the endothelial wall and create a small gap $(\sim 5 \mu \mathrm{m}$ in size $)$ at the EC junction. Upon reaching the subendothelial matrix, protrusions spread into the ECM and the cell squeezes through the EC barrier. (B) During TC transmigration the nucleus the size of the gap increases and crossing of the nucleus takes significantly less time $(\sim 20 \mathrm{~min})$ compared to the whole process of transmigration ( several hours) (90). Following transmigration of the TC adaopts a fully-spread morphology and the junction between the ECs reforms. Schematic diagrams in A indicate the proposed distribution of external forces on a tumour cell during transmigration (such as flow shear, adhesive, tensile, and resistant forces). These forces are generated by and transmitted through several biological complexes such as actomyosin- and integrin-mediated adhesions. Proteinases are also involved in degrading ECM components before/while actin protrusions penetrate. 


\section{Acknowledgments}

EM was recipient of a Wellcome Trust-Massachusetts Institute of Technology Fellowship (WT103883). Funding from the Cancer Research UK [C57744/A22057] and CRUK-UCL Centre Award [C416/A25145] to EM and the US National Cancer Institute (U01 CA202177-01) to RK are gratefully acknowledged. 


\section{References}

1. Spill F, Reynolds DS, Kamm RD, Zaman MH (2016) Impact of the physical microenvironment on tumor progression and metastasis. Curr Opin Biotechnol 40:41-48.

2. Carey SP, D'Alfonso TM, Shin SJ, Reinhart-King CA (2012) Mechanobiology of tumor invasion: Engineering meets oncology. Crit Rev Oncol Hematol 83(2):170183.

3. Shieh AC (2011) Biomechanical Forces Shape the Tumor Microenvironment. Ann Biomed Eng 39(5):1379-1389.

4. Malandrino A, Kamm RD, Moeendarbary E (2017) In Vitro Modeling of Mechanics in Cancer Metastasis. ACS Biomater Sci Eng:acsbiomaterials.7b00041.

5. Moeendarbary E, Harris AR (2014) Cell mechanics: principles, practices, and prospects. Wiley Interdiscip Rev Syst Biol Med 6(5):371-388.

6. Zaman $\mathrm{MH}$ (2013) The role of engineering approaches in analysing cancer invasion and metastasis. Nat Rev Cancer 13(8):596-603.

7. Xu X, Farach-Carson MC, Jia X (2014) Three-dimensional in vitro tumor models for cancer research and drug evaluation. Biotechnol Adv 32(7):1256-1268.

8. Pampaloni F, Reynaud EG, Stelzer EHK (2007) The third dimension bridges the gap between cell culture and live tissue. Nat Rev Mol Cell Biol 8(10):839-45.

9. Kim J Bin (2005) Three-dimensional tissue culture models in cancer biology. Semin Cancer Biol 15(5):365-377.

10. Eglen RM, Randle DH (2015) Drug Discovery Goes Three-Dimensional: Goodbye to Flat High-Throughput Screening? Assay Drug Dev Technol 13(5):262-265.

11. Laschke MW, Menger MD (2017) Life is 3D: Boosting Spheroid Function for Tissue Engineering. Trends Biotechnol 35(2):133-144.

12. Katt ME, Placone AL, Wong AD, Xu ZS, Searson PC (2016) In Vitro Tumor Models: Advantages, Disadvantages, Variables, and Selecting the Right Platform. Front Bioeng Biotechnol 4:12.

13. Tabassum DP, Polyak K (2015) Tumorigenesis: it takes a village. Nat Rev Cancer 15(8):473-483.

14. Wei SC, Yang J (2016) Forcing through Tumor Metastasis: The Interplay between Tissue Rigidity and Epithelial-Mesenchymal Transition. Trends Cell Biol 26(2):111-120.

15. Butcher DT, Alliston T, Weaver VM (2009) A tense situation: forcing tumour progression. Nat Rev Cancer 9(2):108-22.

16. Weis S, Cheresh D (2011) Tumor angiogenesis: molecular pathways and therapeutic targets. Nat Med. Available at: https://www.nature.com/articles/nm.2537 [Accessed July 14, 2017].

17. Santini MT, Rainaldi G Three-dimensional spheroid model in tumor biology. Pathobiology 67(3):148-57.

18. Kunz-Schughart LA, Kreutz M, Knuechel R (1998) Multicellular spheroids: a three-dimensional in vitro culture system to study tumour biology. Int J Exp Pathol 79(1):1-23.

19. Weiswald L-B, Bellet D, Dangles-Marie V (2015) Spherical Cancer Models in Tumor Biology. Neoplasia 17(1):1-15.

20. Nyga A, Cheema U, Loizidou M (2011) 3D tumour models: novel in vitro 
approaches to cancer studies. J Cell Commun Signal 5(3):239-248.

21. Burdett E, Kasper FK, Mikos AG, Ludwig JA (2010) Engineering tumors: a tissue engineering perspective in cancer biology. Tissue Eng Part B Rev 16(3):351-9.

22. Lin R-Z, Chang H-Y, Chang H-Y (2008) Recent advances in three-dimensional multicellular spheroid culture for biomedical research. Biotechnol J 3(9-10):11721184.

23. Thoma CR, Zimmermann M, Agarkova I, Kelm JM, Krek W (2014) 3D cell culture systems modeling tumor growth determinants in cancer target discovery. Adv Drug Deliv Rev 69-70:29-41.

24. LaBarbera D V, Reid BG, Yoo BH (2012) The multicellular tumor spheroid model for high-throughput cancer drug discovery. Expert Opin Drug Discov 7(9):819830.

25. Achilli T-M, Meyer J, Morgan JR (2012) Advances in the formation, use and understanding of multi-cellular spheroids. Expert Opin Biol Ther 12(10):1347-60.

26. Kim J Bin, Stein R, O’Hare MJ (2004) Three-dimensional in vitro tissue culture models of breast cancer - a review. Breast Cancer Res Treat 85(3):281-291.

27. Friedrich J, Ebner R, Kunz-Schughart LA (2007) Experimental anti-tumor therapy in 3-D: Spheroids - old hat or new challenge? Int J Radiat Biol 83(11-12):849871.

28. Brú A, Albertos S, Subiza JL, Ló Pez García-Asenjo J, Brú I (2003) The Universal Dynamics of Tumor Growth. Biophys J 85:2948-2961.

29. Minchinton AI, Tannock IF (2006) Drug penetration in solid tumours. Nat Rev Cancer 6(8):583-592.

30. Vadivelu R, Kamble H, Shiddiky M, Nguyen N-T (2017) Microfluidic Technology for the Generation of Cell Spheroids and Their Applications. Micromachines 8(4):94.

31. Hirschhaeuser F, et al. (2010) Multicellular tumor spheroids: An underestimated tool is catching up again. J Biotechnol 148(1):3-15.

32. Chung S, Sudo R, Vickerman V, Zervantonakis IK, Kamm RD (2010)

Microfluidic Platforms for Studies of Angiogenesis, Cell Migration, and Cell-Cell Interactions. Ann Biomed Eng 38(3):1164-1177.

33. Knowlton S, Onal S, Yu CH, Zhao JJ, Tasoglu S (2015) Bioprinting for cancer research. Trends Biotechnol 33(9):504-513.

34. Jain RK, Martin JD, Stylianopoulos T (2014) The Role of Mechanical Forces in Tumor Growth and Therapy. Annu Rev Biomed Eng 16(1):321-346.

35. Tse JM, et al. (2012) Mechanical compression drives cancer cells toward invasive phenotype. Proc Natl Acad Sci U S A 109(3):911-916.

36. Nia HT, et al. (2016) Solid stress and elastic energy as measures of tumour mechanopathology. Nat Publ Gr 1(November):1-11.

37. Fischbach C, et al. (2007) Engineering tumors with 3D scaffolds. Nat Methods 4(10):855-60.

38. Song H-HG, Park KM, Gerecht S (2014) Hydrogels to model 3D in vitro microenvironment of tumor vascularization. Adv Drug Deliv Rev 79-80:19-29.

39. Ramanujan S, et al. (2002) Diffusion and convection in collagen gels: implications for transport in the tumor interstitium. Biophys $J$ 83(3):1650-60.

40. Tsai H-F, Trubelja A, Shen AQ, Bao G (2017) Tumour-on-a-chip: microfluidic 
models of tumour morphology, growth and microenvironment. $J$ R Soc Interface 14(131). Available at: http://rsif.royalsocietypublishing.org/content/14/131/20170137 [Accessed July 8, 2017].

41. Calvo F, et al. (2013) Mechanotransduction and YAP-dependent matrix remodelling is required for the generation and maintenance of cancer-associated fibroblasts. Nat Cell Biol 15(6):637-646.

42. Labernadie A, et al. (2017) A mechanically active heterotypic E-cadherin/Ncadherin adhesion enables fibroblasts to drive cancer cell invasion. Nat Cell Biol 19(3):224-237.

43. Jeong S-Y, Lee J-H, Shin Y, Chung S, Kuh H-J (2016) Co-Culture of Tumor Spheroids and Fibroblasts in a Collagen Matrix-Incorporated Microfluidic Chip Mimics Reciprocal Activation in Solid Tumor Microenvironment. PLoS One 11(7):e0159013.

44. DelNero P, et al. (2015) 3D culture broadly regulates tumor cell hypoxia response and angiogenesis via pro-inflammatory pathways. Biomaterials 55:110-118.

45. Funamoto K, et al. (2012) A novel microfluidic platform for high-resolution imaging of a three-dimensional cell culture under a controlled hypoxic environment. Lab Chip 12(22):4855-63.

46. Madsen CD, et al. (2015) Hypoxia and loss of PHD2 inactivate stromal fibroblasts to decrease tumour stiffness and metastasis. EMBO Rep 16(10):1394-1408.

47. Koumoutsakos P, Pivkin I, Milde F (2013) The Fluid Mechanics of Cancer and Its Therapy. Annu Rev Fluid Mech 45(1):325-355.

48. Rieger H, Welter M (2015) Integrative models of vascular remodeling during tumor growth. Wiley Interdiscip Rev Syst Biol Med 7(3):113-129.

49. Nguyen D-HT, et al. (2013) Biomimetic model to reconstitute angiogenic sprouting morphogenesis in vitro. Proc Natl Acad Sci U S A 110(17):6712-7.

50. Galie PA, et al. (2014) Fluid shear stress threshold regulates angiogenic sprouting. Proc Natl Acad Sci U S A 111(22):7968-73.

51. Boldock L, Wittkowske C, Perrault CM (2017) Microfluidic traction force microscopy to study mechanotransduction in angiogenesis. Microcirculation 24(5):e12361.

52. Bray LJ, Werner C (2017) Evaluation of Three-Dimensional in Vitro Models to Study Tumor Angiogenesis. ACS Biomater Sci Eng:acsbiomaterials.7b00139.

53. Song JW, Bazou D, Munn LL (2012) Anastomosis of endothelial sprouts forms new vessels in a tissue analogue of angiogenesis. Integr Biol (Camb) 4(8):857-62.

54. Amann A, Zwierzina M, Koeck S, Gamerith G (2017) Development of a 3D angiogenesis model to study tumour-endothelial cell interactions and the effects of anti-angiogenic drugs. Scientific. Available at: https://www.ncbi.nlm.nih.gov/pmc/articles/PMC5462801/ [Accessed July 15, 2017].

55. Correa de Sampaio P, et al. (2012) A Heterogeneous In Vitro Three Dimensional Model of Tumour-Stroma Interactions Regulating Sprouting Angiogenesis. PLoS One 7(2):e30753.

56. Upreti M, et al. (2011) Tumor-Endothelial Cell Three-dimensional Spheroids: New Aspects to Enhance Radiation and Drug Therapeutics. Transl Oncol 
4(6):365-IN3.

57. Seano G, et al. (2013) Modeling human tumor angiogenesis in a three-dimensional culture system. Blood 121(21):e129-37.

58. Nguyen DT, Fan Y, Akay YM, Akay M (2016) Investigating Glioblastoma Angiogenesis Using A 3D in Vitro GelMA Microwell Platform. IEEE Trans Nanobioscience 15(3):289-293.

59. Taubenberger A V., et al. (2016) 3D extracellular matrix interactions modulate tumour cell growth, invasion and angiogenesis in engineered tumour microenvironments. Acta Biomater 36:73-85.

60. Bray LJ, et al. (2015) Multi-parametric hydrogels support 3D in vitro bioengineered microenvironment models of tumour angiogenesis. Biomaterials 53:609-620.

61. Stroka KM, Konstantopoulos K (2014) Physical biology in cancer. 4. Physical cues guide tumor cell adhesion and migration. Am J Physiol Cell Physiol 306(2):C98-C109.

62. Polacheck WJ, Li R, Uzel SGM, Kamm RD (2013) Microfluidic platforms for mechanobiology. Lab Chip 13(12):2252-67.

63. Kramer N, et al. (2013) In vitro cell migration and invasion assays. Mutat Res Mutat Res 752(1):10-24.

64. Balzer EM, et al. (2012) Physical confinement alters tumor cell adhesion and migration phenotypes. FASEB J 26(10):4045-4056.

65. Tong Z, et al. (2012) Chemotaxis of Cell Populations through Confined Spaces at Single-Cell Resolution. PLoS One 7(1):e29211.

66. Stroka KM, et al. (2014) Water Permeation Drives Tumor Cell Migration in Confined Microenvironments. Cell 157(3):611-623.

67. Moeendarbary E, et al. (2013) The cytoplasm of living cells behaves as a poroelastic material. Nat Mater 12(3):253-61.

68. Charras G, Paluch E (2008) Blebs lead the way: how to migrate without lamellipodia. Nat Rev Mol Cell Biol 9(9):730-736.

69. Denais CM, et al. (2016) Nuclear envelope rupture and repair during cancer cell migration. Science (80- ) 352(6283):353-358.

70. Charras G, Sahai E (2014) Physical influences of the extracellular environment on cell migration. Nat Rev Mol Cell Biol 15(12):813-824.

71. Chaudhuri O, et al. (2014) Extracellular matrix stiffness and composition jointly regulate the induction of malignant phenotypes in mammary epithelium. Nat Mater 13(10):970-8.

72. Polacheck WJ, Charest JL, Kamm RD (2011) Interstitial flow influences direction of tumor cell migration through competing mechanisms. Proc Natl Acad Sci U S A 108(27):11115-20.

73. Huang YL, Tung C-K, Zheng A, Kim BJ, Wu M (2015) Interstitial flows promote amoeboid over mesenchymal motility of breast cancer cells revealed by a three dimensional microfluidic model. Integr Biol (Camb) 7(11):1402-11.

74. Polacheck WJ, German AE, Mammoto A, Ingber DE, Kamm RD (2014) Mechanotransduction of fluid stresses governs 3D cell migration. Proc Natl Acad Sci U S A 111(7):2447-52.

75. Li R, et al. (2017) Macrophage-secreted TNF $\alpha$ and TGF $\beta 1$ influence migration 
speed and persistence of cancer cells in 3D tissue culture via independent pathways. Cancer Res 77(2):279-290.

76. Piotrowski-Daspit AS, Tien J, Nelson CM (2016) Interstitial fluid pressure regulates collective invasion in engineered human breast tumors via Snail, vimentin, and E-cadherin. Integr Biol (Camb) 8(3):319-331.

77. Agastin S, Giang U-BT, Geng Y, Delouise LA, King MR (2011) Continuously perfused microbubble array for 3D tumor spheroid model. Biomicrofluidics 5(2):24110.

78. Sakai Y, et al. (2014) Detachably assembled microfluidic device for perfusion culture and post-culture analysis of a spheroid array. Biotechnol J 9(7):971-979.

79. Anderberg C, et al. (2013) Deficiency for endoglin in tumor vasculature weakens the endothelial barrier to metastatic dissemination. 210(3). doi:10.1084/jem.20120662.

80. Roussos ET, et al. (2011) Mena invasive (MenaINV) promotes multicellular streaming motility and transendothelial migration in a mouse model of breast cancer. J Cell Sci 124(Pt 13):2120-31.

81. Brábek J, Mierke CT, Rösel D, Veselý P, Fabry B (2010) The role of the tissue microenvironment in the regulation of cancer cell motility and invasion. 1-8.

82. Mierke CT (2011) Cancer cells regulate biomechanical properties of human microvascular endothelial cells. J Biol Chem 286(46):40025-37.

83. Chrobak KM, Potter DR, Tien J (2006) Formation of perfused, functional microvascular tubes in vitro. Microvasc Res 71(3):185-96.

84. Zheng Y, et al. (2012) In vitro microvessels for the study of angiogenesis and thrombosis. Proc Natl Acad Sci U S A 109(24):9342-7.

85. Kolesky DB, Homan K a, Skylar-Scott M a, Lewis J a (2016) Three-dimensional bioprinting of thick vascularized tissues. Proc Natl Acad Sci U S A 113(12):3179_ 84.

86. Shin MK, Kim SK, Jung H (2011) Integration of intra- and extravasation in one cell-based microfluidic chip for the study of cancer metastasis. Lab Chip 11(22):3880-7.

87. Ehsan SM, et al. (2015) A three-dimensional in vitro model of tumor cell intravasation. Integr Biol 6(6):603-610.

88. Khuon S, et al. (2010) Myosin light chain kinase mediates transcellular intravasation of breast cancer cells through the underlying endothelial cells: a three-dimensional FRET study. J Cell Sci 123(Pt 3):431-40.

89. Whisler J a, Chen MB, Kamm RD (2014) Control of perfusable microvascular network morphology using a multiculture microfluidic system. Tissue Eng Part C Methods 20(7):543-52.

90. Chen MB, Whisler JA, Jeon JS, Kamm RD (2013) Mechanisms of tumor cell extravasation in an in vitro microvascular network platform. Integr Biol 5(10):1262-1271.

91. Díaz de la Loza MC, et al. (2017) Laminin Levels Regulate Tissue Migration and Anterior-Posterior Polarity during Egg Morphogenesis in <em $>$ Drosophila</em $>$. Cell Rep 20(1):211-223.

92. Yeon JH, Ryu HR, Chung M, Hu QP, Jeon NL (2012) In vitro formation and characterization of a perfusable three-dimensional tubular capillary network in 
microfluidic devices. Lab Chip 12(16):2815-22.

93. Bockhorn M, Jain RK, Munn LL (2007) Active versus passive mechanisms in metastasis: do cancer cells crawl into vessels, or are they pushed? Lancet Oncol 8(5):444-448.

94. Wyckoff JB, et al. (2007) Direct visualization of macrophage-assisted tumor cell intravasation in mammary tumors. Cancer Res 67(6):2649-2656.

95. Condeelis J, Segall JE (2003) Intravital imaging of cell movement in tumours. Nat Rev Cancer 3(12):921-30.

96. Zervantonakis IK, et al. (2012) Three-dimensional microfluidic model for tumor cell intravasation and endothelial barrier function. Proc Natl Acad Sci 109(34):13515-13520.

97. Chiang SPH, Cabrera RM, Segall JE (2016) Tumor Cell Intravasation. Am J cell Physiol Physiol 311(1):C1-C14.

98. Reymond N, d'Água BB, Ridley AJ (2013) Crossing the endothelial barrier during metastasis. Nat Rev Cancer 13(12):858-870.

99. Kraning-Rush CM, et al. (2012) Cellular Traction Stresses Increase with Increasing Metastatic Potential. PLoS One 7(2):e32572.

100. Li Y-H, Zhu C (1999) A modified Boyden chamber assay for tumor cell transendothelial migration in vitro. Clin Exp Metastasis 17(5):423-429.

101. Wong AD, Searson PC (2014) Live-cell imaging of invasion and intravasation in an artificial microvessel platform. Cancer Res:4937-4946.

102. Nashimoto Y, et al. (2017) Integrating perfusable vascular networks with a threedimensional tissue in a microfluidic device. Integr Biol 9(6):506-518.

103. Psaila B, Lyden D (2009) The metastatic niche: adapting the foreign soil. 9(APrIl):285-293.

104. Crissman JD, Hatfield JS, Menter DG, Sloane B, Honn KY (1988) Morphological Study of the Interaction of Intra vascular Tumor Cells with Endothelial Cells and Subendothelial Matrix1. 4065-4072.

105. Bussard KM, Gay C V, Mastro AM (2008) The bone microenvironment in metastasis; what is special about bone? Cancer Metastasis Rev 27(1):41-55.

106. Fidler IJ (2011) The role of the organ microenvironment in brain metastasis. Semin Cancer Biol 21(2):107-12.

107. Chambers AF, Groom AC, MacDonald IC (2002) Dissemination and growth of cancer cells in metastatic sites. Nat Rev Cancer 2(8):563-72.

108. Miles FL, Pruitt FL, van Golen KL, Cooper CR (2008) Stepping out of the flow: capillary extravasation in cancer metastasis. Clin Exp Metastasis 25(4):305-324.

109. Strell C, et al. (2012) Norepinephrine promotes the $\beta 1$-integrin-mediated adhesion of MDA-MB-231 cells to vascular endothelium by the induction of a GRO $\alpha$ release. Mol Cancer Res 10(2):197-207.

110. Barthel SR, et al. (2013) Definition of Molecular Determinants of Prostate Cancer Cell Bone Extravasation De fi nition of Molecular Determinants of Prostate Cancer Cell. 942-952.

111. Matrone MA, Whipple RA, Balzer EM, Martin SS (2010) Microtentacles tip the balance of cytoskeletal forces in circulating tumor cells. Cancer Res 70(20):773741.

112. Hiratsuka S, Watanabe A, Aburatani H, Maru Y (2006) Tumour-mediated 
upregulation of chemoattractants and recruitment of myeloid cells predetermines lung metastasis. Nat Cell Biol 8(12):1369-75.

113. Auguste P, et al. (2007) The Host Inflammatory Response Promotes Liver Metastasis by Increasing Tumor Cell Arrest and Extravasation. Am J Pathol 170(5):1781-1792.

114. Draffin JE, Mcfarlane S, Hill A, Johnston PG, Waugh DJJ (2004) CD44 Potentiates the Adherence of Metastatic Prostate and Breast Cancer Cells to Bone Marrow Endothelial Cells. 5702-5711.

115. Wang H, Hung Y, Su C, Peng S, Guo Y (2005) CD44 Cross-linking induces integrin-mediated adhesion and transendothelial migration in breast cancer cell line by up-regulation of LFA-1 ( a L h 2 ) and VLA-4 ( a 4 h 1 ). 304:116-126.

116. Chen MB, Lamar JM, Li R, Hynes RO, Kamm RD (2016) Elucidation of the roles of tumor integrin ss1 in the extravasation stage of the metastasis cascade. Cancer Res:1-13.

117. Labelle M, Begum S, Hynes RO (2011) Direct signaling between platelets and cancer cells induces an epithelial-mesenchymal-like transition and promotes metastasis. Cancer Cell 20(5):576-90.

118. Voura EB, et al. (2013) Proteolysis during tumor cell extravasation in vitro: metalloproteinase involvement across tumor cell types. PLoS One 8(10):e78413.

119. Zaman MH, et al. (2006) Migration of tumor cells in 3D matrices is governed by matrix stiffness along with cell-matrix adhesion and proteolysis. Proc Natl Acad Sci U S A 103(29):10889-94.

120. Leong HS, et al. (2014) Invadopodia are required for cancer cell extravasation and are a therapeutic target for metastasis. Cell Rep 8(5):1558-70.

121. Ganguly KK, Pal S, Moulik S, Chatterjee A (2013) Integrins and metastasis. (June):251-261.

122. Palumbo JS, et al. (2017) Platelets and fibrin ( ogen ) increase metastatic potential by impeding natural killer cell - mediated elimination of tumor cells. 105(1):178186.

123. Erpenbeck L, Scho MP (2017) Review article Deadly allies : the fatal interplay between platelets and metastasizing cancer cells. 115(17):3427-3437.

124. Liu Y, et al. (2011) Tissue Factor - Activated Coagulation Cascade in the Tumor Microenvironment Is Critical for Tumor Progression and an Effective Target for Therapy. 71(20):6492-6503.

125. Schumacher D, Strilic B, Sivaraj KK, Wettschureck N, Offermanns S (2013) Platelet-derived nucleotides promote tumor-cell transendothelial migration and metastasis via P2Y2 receptor. Cancer Cell 24(1):130-7.

126. Borsig L, Wong R, Hynes RO, Varki NM, Varki A (2001) Synergistic effects of Land $\mathrm{P}$-selectin in facilitating tumor metastasis can involve non-mucin ligands and implicate leukocytes as enhancers of metastasis. (9).

127. Granot Z, et al. (2011) Tumor entrained neutrophils inhibit seeding in the premetastatic lung. Cancer Cell 20(3):300-314.

128. Huh SJ, Liang S, Sharma A, Dong C, Robertson GP (2010) Transiently entrapped circulating tumor cells interact with neutrophils to facilitate lung metastasis development. Cancer Res 70(14):6071-6082.

129. Labelle M, Begum S, Hynes RO (2014) Platelets guide the formation of early 
metastatic niches. Proc Natl Acad Sci U S A 111(30):E3053-61.

130. Bergers G, Song S (2005) The role of pericytes in blood-vessel formation and maintenance 1. doi:10.1215/S1152851705000232.

131. Zhang P, Goodrich C, Fu C, Dong C (2014) Melanoma upregulates ICAM-1 expression on endothelial cells through engagement of tumor CD44 with endothelial E-selectin and activation of a PKC $\alpha$-p38-SP-1 pathway. FASEB J 28(11):4591-4609.

132. Chen MB, et al. (2017) On-chip human microvasculature assay for visualization and quantification of tumor cell extravasation dynamics. Nat Protoc 12(5):865880 . 